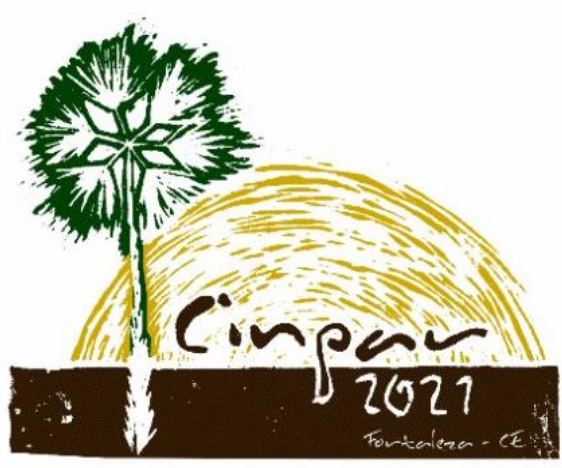

XVII Congresso Internacional sobre Patologia e

Reabilitação das Construções

XVII Congreso Internacional sobre Patología y Rehabilitación de las Construcciones

XVII International Conference on Pathology and Constructions Rehabilitation

FORTALEZA (Brasil), 3 a 5 de junho de 2021

https://doi.org/10.4322/CINPAR.2021.035

\title{
Abordagem estatística da produção científica internacional sobre medidas de mitigação da Reação Álcali-Sílica (RAS)
}

\section{Statistical approach of international scientific production on mitigation measures of the Alkali-Silica Reaction (ASR)}

\author{
Aloísio Ângelo dos Santos Neto ${ }^{1}$, Kelvya Maria de Vasconcelos Moreira ${ }^{2}$, Ênio Pontes de Deus ${ }^{3}$, Antônio \\ Eduardo Bezerra Cabral ${ }^{4}$

\footnotetext{
${ }^{1}$ Universidade Estadual Vale do Acaraú, Sobral, Brasil, aloisioneto432@gmail.com

2 Universidade Estadual Vale do Acaraú, Sobral, Brasil, kelvyamoreira@gmail.com

${ }^{3}$ Universidade Federal do Ceará, Fortaleza, Brasil, epontes@ufc.br

${ }^{4}$ Universidade Federal do Ceará, Fortaleza, Brasil, eduardo.cabral@ufc.br
}

\begin{abstract}
Resumo: A pesquisa por referências bibliográficas, parte fundamental deste artigo, exige cautela durante a busca, seleção e análise dos dados. Compõem este processo a análise bibliométrica e a revisão sistemática das publicações científicas a fim de fundamentar quantitativamente a pesquisa. Tais métodos de busca são comuns nas áreas de Engenharia e Saúde. Neste sentido, o presente artigo objetiva averiguar as publicações científicas internacionais que abordam medidas de mitigação da Reação Álcali-Sílica (RAS) a partir da utilização de técnicas bibliométricas e da metodologia de seleção Proknow-C nas bases de dados SciELO, Web of Science, Scopus e ScienceDirect. A RAS é um tipo de manifestação patológica endógena que ocorre em estruturas de concreto pela reação química entre a sílica amorfa ou mal cristalizada e hidróxidos alcalinos. Esta reação é a variação mais frequente da Reação Álcali-Agregado (RAA), além de ser a mais pesquisada e bem compreendida. Ela tem como produto um gel higroscópico geralmente expansivo que ocupa os poros dos compósitos cimentícios. A quantidade de gel e sua magnitude dependem, principalmente, da temperatura e da quantidade de cimento consumido. Durante décadas essa anomalia foi compreendida como incidente em grandes obras hídricas, como barragens de concreto e pilares de pontes, entretanto, devido ao aumento de casos diagnosticados em estruturas menores, como pavimentos e fundações de edifícios, passou-se a observar um maior entendimento e preocupação da comunidade científica com essa reação. Logo, tem ocorrido uma ampla disseminação dessas informações por meio de publicações de artigos em periódicos e anais de eventos, o que motivou a realização deste estudo. Os dados foram coletados após a análise de 204 artigos publicados entre os anos de 2004 e 2020. A interpretação dos resultados permitiu confirmar o expressivo crescimento da produção científica, além de determinar os países que mais publicaram e as práticas mais pesquisadas sobre a mitigação da RAS.
\end{abstract}

Palavras-chave: reação álcali-sílica; mitigação; análise estatística.

Abstract: The search for bibliographic references, a fundamental part of this article, requires caution when searching, selecting and analyzing data. This process comprises bibliometric analysis and systematic review of scientific publications in order to substantiate the research quantitatively. Such search methods are common in the areas of Engineering and Health. In this sense, this article aims to investigate international scientific publications that address mitigation measures of the Alkali-Silica Reaction (ASR) from the use of bibliometric techniques and the selection methodology Proknow-C in the SciELO, Web of Science, Scopus 
and ScienceDirect databases. ASR is a type of endogenous pathological manifestation that occurs in concrete structures by the chemical reaction between amorphous or poorly crystallized silica and alkaline hydroxides. This reaction is the most frequent variation of the Alkali-Aggregate Reaction (AAR), in addition to being the most researched and well understood. It has as a product a hygroscopic gel generally expansive that occupies the pores of cementitious composites. The amount of gel and its magnitude depends mainly on the temperature and the amount of cement consumed. For decades, this anomaly was understood as an incident in large water works, such as concrete dams and pillars of bridges. However, due to the increase in cases diagnosed in smaller structures, such as pavements and foundations of buildings, a greater understanding and concern of the scientific community with this reaction. Therefore, there has been a wide dissemination of this information through the publication of articles in periodicals and annals of events, which motivated the realization of this study. The data were collected after the analysis of 204 articles published between the years 2004 and 2020. The interpretation of the results allowed to confirm the expressive growth of scientific production, in addition to determining the countries that published the most and the most researched practices on the mitigation of ASR.

Keywords: alkali-silica reaction; mitigation; statistical analysis.

\section{Introdução}

A Reação Álcali-Sílica (RAS) é uma manifestação patológica de alta deterioração que ameaça a durabilidade das estruturas de concreto. Caracterizada como sendo de longo prazo, a RAS é uma reação química entre certas formas de sílica reativa, que por possuírem estruturas um pouco desordenadas se tornam instáveis num meio de elevado $\mathrm{pH}$, e os íons alcalinos ( $\mathrm{Na}+$ e $\mathrm{K}+$ ) e hidróxilos $(\mathrm{OH}-)$ presentes na solução intersticial da pasta de cimento, produzindo um gel de silicato alcalinohidratado que se expande com a absorção de água causandofissuras e, consequente, deterioração da estrutura. Portanto, a RAS é um processo mecânico em escala de engenharia de origem química. Essa reação normalmente necessita de um longo período para se manifestar e quando se manifesta na sua forma nociva, porporciona a expansão do concreto, surgimento de fissuras e diminuição da sua resistência química e mecânica (HERNÁNDEZ-CRUZ et al, 2016).

A comunidade científica segrega em duas a Reação Álcali-Agregado (RAA): Reação Álcali-Carbonato (RAC), dada como uma reação entre álcalis e agregados de carbonato; e Reação Álcali-Sílica (RAS), dada como uma reação entre álcalis e agregados contendo sílica reativa. Ainda existe uma terceiravariação dessa reação, a Reação Álcali-Silicato, mas essa é considerada uma variação mais lenta da RAS. A RAS é amanifestação mais pesquisada pelo meio técnico devido a sua maior complexidade e rápido desenvolvimento, além de, normalmente, ser o principal processo responsável pela deterioração das estruturas devido à presença mais frequente de formas reativas de sílica, como opala, tridimita, calcedônia e quartzo deformado, nos agregados usados no concreto (RIBEIRO et al, 2012).

Ao longo dos anos as principais manifestações dessa anomalia foram verificadas em grandes obras hidráulicas, estruturas submetidas à grande volume de compósitos cimentícios e elevada umidade. Hoje, já se sabe que esta reação também se manifesta em estruturas menores, como pavimentos e fundações de edifícios residenciais. Vários países já relataram graves ocorrências de RAS. Em alguns casos a deterioração da estrutura foi incorretamente diagnosticada, excluindo a reação com álcalis; em outros, não foi diagnosticada RAS destrutiva, mas foi constatada a presença de agregados reativos. 0 aumento de registros de estruturas afetadas por reação álcali-sílica se deve ao maior entendimento sobre essa reação e a preocupação com suas consequências. Mesmo assim, ainda há muitos casos com diagnóstico tardio, onde a reação já se encontra em estado avançado, dificultando o tratamento e recuperação. Com isso vêm sendo desenvolvidas várias pesquisas científicas sobre a reação álcali-sílica, a fim de compreender sua ação e criar meios de mitigação ou prevenção (ZAPALA-SLAWETA; OWSIAK, 2018).

Mediante a crescente descoberta de estruturas de concreto que apresentam sinais graves de deterioração por RAS, o atual trabalho objetiva analisar as publicações científicas internacionais sobre medidas de mitigação a fim de mapear o avanço das pesquisas pelo mundo e estabelecer quais técnicas mais utilizadas e as que tiveram melhor resultado. Far-se-á uso do sistema de coleta Proknow-C (Knowledge Development Process - Constructivist), que é um método de revisão sistemático desenvolvido e patenteado pela 
Universidade Federal de Santa Catarina (AFONSO et al, 2011) para seleção dos autores, dos artigos e dos periódicos destacáveis, possibilitando a criação do embasamento teórico. Também far-se-á uso da bibliometria, que é uma ferramenta de análise quantitativa, por se basear na contagem de artigos científicos, citações e patentes para mensurar a contribuição das publicações em determinadas áreas. Soares e seus colegas (2015) afirmam que, dependendo da finalidade do estudo bibliométrico, os dados podem ser tanto o texto que compõe a publicação como os elementos presentes em registros sobre publicações extraídos de base de dados bibliográficos, como nome de autores, título, fonte, idioma, palavras-chave, classificação e citações. Os autores ainda ressaltam que identificar tendências de crescimento do conhecimento, autores e instituições mais produtivas, periódicos mais usados e dispersão e obsolescências de campos científicos são algumas das contribuições da bibliometria.

\section{Materiais e Métodos}

A metodologia utilizada na pesquisa foi dividida em etapas. Inicialmente foram estabelecidos seus métodos de condução, definindo-se as bases de dados utilizadas, os filtros de busca, a metodologia da construção do arcabouço bibliográfico e os algoritmos de busca mais pertinentes. A etapa seguinte consistiu da busca dos artigos em todas as bases de dados selecionadas, utilizando os algoritmos definidos. A próxima etapa baseou-se na coleta dos indicadores bibliométricos dos artigos selecionados e montagem de gráficos e tabelas desses dados. E como etapa final fez-se a análise e interpretação dos resultados obtidos na pesquisa. Para a realização deste estudo optou-se pela utilização de 04 bases de dados: (1) SciELO, (2) ScienceDirect, (3) Scopus e (4) Web of Science (WoS), pesquisando em todas elas as publicações científicas que abordacem a mitigação da Reação Álcali-Sílica. Fizeram parte da busca os artigos publicados em periódicos e os anais de congressos indexados nas bases de dados citadas na área do conhecimento da engenharia civil. Vale ressaltar que compuseram o portfólio bibliográfico apenas as publicações com acesso livre nas bases de dados.

Definiu-se o interstício de 2004 a 2020 como intervalo de tempo das buscas. A escolha do marco inicial foi baseada no ano do colapso do Edifício Areia Branca, localizado na zona sul da Região Metropolitana do Recife, Pernambuco, Brasil, evento que culminou na elaboração da primeira edição da normativa brasileira sobre a reação expansiva, a ABNT NBR 15577:2008.

Durante as buscas nas bases de dados foram utilizados os seguintes algorítimos: "reação álcali-sílica", "mitigação", "RAS", "agregado reativo" e "álcalis do cimento". Após a catalogação dos artigos, foram lidos os resumos e selecionados aqueles artigos que melhor se enquadravam no objetivo principal desta pesquisa. As referências dos artigos encontrados foram exportadas para um gerenciador online de referências bibliográficas (EndNote) a fim de verificar a existência de artigos repetidos, uma vez que foi utilizado mais de um banco de dados. Posteriormente, foi feita a quantificação dos indicadores bibliométricos.

Os indicadores bibliométricos utilizados para a construção dos gráficos e tabelas foram: ano de publicação, autor, país, universidade, periódico e método de mitigação da RAS. Foram coletados 407 artigos científicos somados de todas as bases de dados e, após a leitura de seus resumos, verificou-se que apenas 204 artigos se encaixavam diretamente no tema abordado.

\section{Resultados e discussões}

Pela Figura 1 observa-se que a produção de artigos com a abordagem sobre medidas de mitigação da RAS teve um aumento significativo a partir de 2017, com destaque para o pico ocorrido em 2019 (21 artigos). A busca pelos artigos foi feita entre os meses de julho e novembro de 2020, por isso o valor referente a este ano pode sofrer alterações, estima-se que o número nesse ano ultrapasse 20 publicações.Vale ressaltar que os artigos podem ter associação com mais de uma instituição, pois os 204 artigos foram vinculados a 326 instituições, uma média de 1,6 instituições por artigo. Das 326 instituições vinculadas às publicações, 9 (2,8\%) são Agências Governamentais, 21 são Empresas (6,4\%), 22 são Institutos de Pesquisa (6,7\%), 8 são Universidades Privadas (2,5\%) e 266 são Universidades Públicas (81,6\%). 


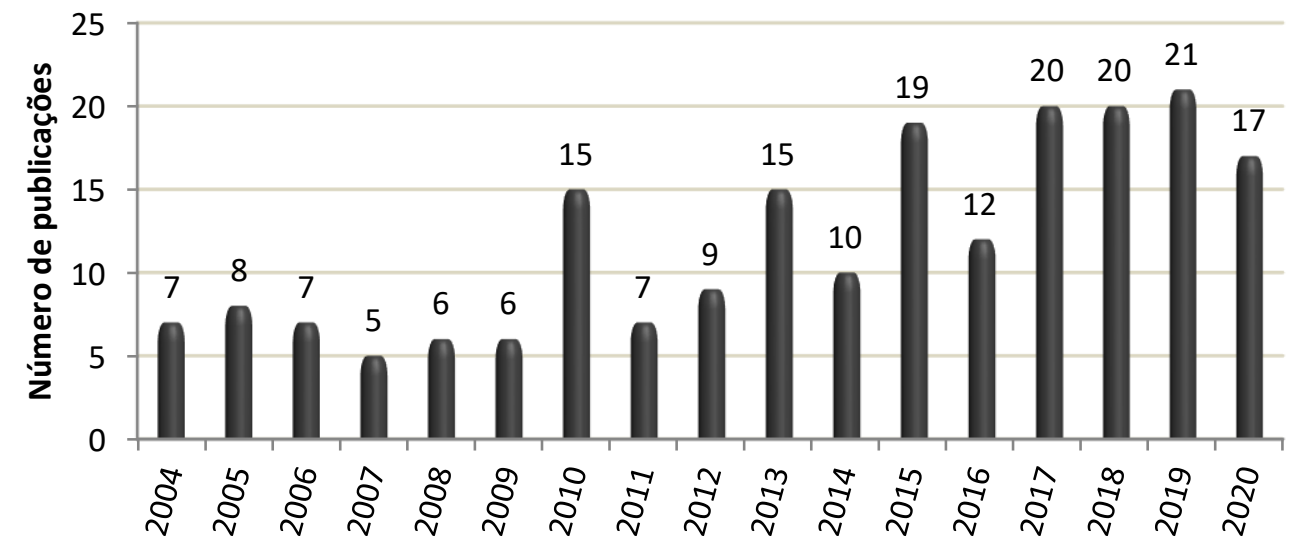

Figura 1 - Evolução da produção científica internacional sobre as medidas de mitigação da Reação Álcali-Sílica no período de 2004 a 2020

As instituições que mais publicaram sobre mitigação da RAS, como ilustrado na Tabela 1, foram a Kielce University of Technology (13 publicações), localizada na Polônia; e as universidades Norte-Americanas Clemson University (Carolina do Norte) e University of Texas (Austin), com 11 publicações cada. A Tabela 2 apresenta os autores que mais publicaram: em primeiro está a Justyna Zapala-Slaweta (12 publicações), professora assistente do Departamento de Tecnologia e Organização da Construção da Kielce University of Technology, que pesquisou sobre os tratamentos com compostos de lítio e materiais cimentícios suplementares, principalmente o nitrato de lítio e o metacaulim; seguida por Prasada Rao Rangaraju (10 publicações), professor do Departamento de Engenharia Civil da Clemson University, cujas pesquisas foram voltadas para o tratamento com materiais cimentícios suplementares, principalmente a cinza volante; e Benoît Fournier (9 publicações), professor titular do departamento de geologia e engenharia geológica da Université Laval, que pesquisou sobre a mitigação da RAS utilizando materiais cimentícios suplementares e com compostos de lítio, principalmente e cinza volante nitrato de lítio.

Tabela 1 - Ranking das 10 instituições com mais publicações sobre a mitigação da Reação Álcali-Sílica no período de 2004 a 2020

\begin{tabular}{c|c|c|c}
\hline Instituição & País da Instituição & Artigos & $\mathbf{\%}$ \\
\hline Kielce University of Technology & Polônia & 13 & $6,4 \%$ \\
Clemson University & Estados Unidos & 11 & $5,4 \%$ \\
University of Texas at Austin & Estados Unidos & 11 & $5,4 \%$ \\
University of New Brunswick & Estados Unidos & 9 & $4,4 \%$ \\
Université Laval & Canadá & 8 & $3,9 \%$ \\
Pennsylvania State University & Estados Unidos & 6 & $2,9 \%$ \\
University of California & Estados Unidos & 6 & $2,9 \%$ \\
Ege University & Turquia & 5 & $2,5 \%$ \\
Nanjing Normal University & China & 5 & $2,5 \%$ \\
University of Arkansas / Oregon State University & Estados Unidos & 4 & $2,0 \%$ \\
\hline
\end{tabular}

Tabela 2 - Ranking dos 5 autores com mais publicações sobre a mitigação da Reação Álcali-Sílica no período de 2004 a 2020

\begin{tabular}{|c|c|c|c|c|}
\hline Autor & Instituição & País da Instituição & Artigos & $\%$ \\
\hline Justyna Zapala-Slaweta & $\begin{array}{l}\text { Kielce University of } \\
\text { Technology }\end{array}$ & Polônia & 12 & $5,9 \%$ \\
\hline Prasada Rao Rangaraju & Clemson University & Estados Unidos & 10 & $4,9 \%$ \\
\hline Benoît Fournier & Université Laval & Canadá & 9 & $4,4 \%$ \\
\hline Michael D. A. Thomas & University of New Brunswick & Estados Unidos & 9 & $4,4 \%$ \\
\hline Zdzislawa Owsiak & $\begin{array}{c}\text { Kielce University of } \\
\text { Technology }\end{array}$ & Polônia & 9 & $4,4 \%$ \\
\hline
\end{tabular}


A Tabela 3 ilustra os países com mais publicações sobre o tema. Em primeiro estão os Estados Unidos (63 autorias), além de seis universidades entre as dez que mais publicaram e dois autores entre os cinco maiores publicadores. Em seguida vem a China, Turquia, Canadá, Polônia, França, Austrália, Japão e Suíça. A Tabela 4 ilustra os principais periódicos utilizados para publicação sobre a mitigação da Reação ÁlcaliSílica, evidenciando a fonte de disseminação mais utilizada pelos pesquisadores. O periódico com mais artigos publicados sobre o tema é o Construction and Building Materials, da Elsevier, com 32 artigos (15,69\% do total da produção analisada). Dentre os 10 periódicos com mais artigos publicados no tema, cinco pertencem à área de pesquisa sobre Materiais (Construction and Building Materials, Aci Materials Journal, Journal of Materials in Civil Engineering, Materials and Structures e Cement Wapno Beton), quatro sobre Concreto (Cement and Concrete Research, Cement \& Concrete Composites, Magazine of Concrete Research e Structural Concrete) e um sobre Meio Ambiente (Journal of Cleaner Production).

Tabela 3 - Ranking dos 10 países com mais publicações sobre a mitigação da Reação Álcali-Sílica no período de 2004 a 2020

\begin{tabular}{c|c|c}
\hline País & Artigos & $\mathbf{\%}$ \\
\hline Estados Unidos & 63 & $30,9 \%$ \\
China & 36 & $17,6 \%$ \\
Turquia & 21 & $10,3 \%$ \\
Canadá & 20 & $9,8 \%$ \\
Polônia & 16 & $7,8 \%$ \\
França & 10 & $4,9 \%$ \\
Austrália & 9 & $4,4 \%$ \\
Brasil & 8 & $3,9 \%$ \\
Japão & 7 & $3,4 \%$ \\
Suíça & 6 & $2,9 \%$ \\
\hline
\end{tabular}

Tabela 4 - Ranking dos 10 periódicos com mais publicações sobre a mitigação da Reação Álcali-Sílica no período de 2004 a 2020

\begin{tabular}{c|c|c}
\hline Periódico & Artigos & $\mathbf{\%}$ \\
\hline Construction and Building Materials & 32 & $15,7 \%$ \\
Cement and Concrete Research & 31 & $15,2 \%$ \\
Aci Materials Journal & 12 & $5,9 \%$ \\
Cement \& Concrete Composites & 10 & $4,9 \%$ \\
Journal of Materials in Civil Engineering & 9 & $4,4 \%$ \\
Materials and Structures & 6 & $2,9 \%$ \\
Cement Wapno Beton & 5 & $2,5 \%$ \\
Journal of Cleaner Production & 4 & $2,0 \%$ \\
Magazine of Concrete Research & 3 & $1,5 \%$ \\
Structural Concrete & 3 & $1,5 \%$ \\
\hline
\end{tabular}

As medidas preventivas da RAS se concentram na eliminação dos fatores que contribuem para os processos destrutivos ou alterar o ambiente químico necessário para o desenvolvimento da reação. Compõe este processo: o uso de agregados não reativos, cimentos de baixo teor alcalino, aditivos minerais ou misturas químicas, incorporados durante a mistura. Baixar a umidade relativa do concreto abaixo de $80 \%$ é outra medida para limitar a reação destrutiva (MENÉNDEZ et al, 2020). Sua prevenção é bem simples quando comparada as técnicas de recuperação disponíveis, que normalmente são onerosas e pouco eficazes. A Tabela 5 evidencia a segregação dos métodos utilizados para mitigar a RAS abordados nos artigos selecionados. $\mathrm{O}$ tratamento mais abordado é a utilização de materiais cimentícios suplementares, com 81 publicações, utilizando principalmente cinzas volantes das classes C e F, metacaulim, sílica ativa e escória granulada de alto forno. Tais materiais atuam na dissolução do cimento Portland, diminuindo os hidróxidos de cálcio e alcalinos liberados para a pasta hidratada do cimento, além de reduzirem a porosidade do 
compósito. Estes materiais tem sido empregados em muitas construções de concreto a fim de prevenir a RAS, porém nem todos os materiais cimentícios suplementares são igualmente efetivos neste papel e a quantidade deles precisa ser criteriosamente avaliada caso a caso, pois podem se tornar não aceitáveis por gerarem falsos resultados.

Tabela 5 - Distribuição das medidas de mitigação da Reação Álcali-Sílica no período de 2004 a 2020

\begin{tabular}{c|c|c}
\hline Medida de mitigação utilizada & Artigos & $\%$ \\
\hline Tratamento com materiais cimentícios suplementares (SCMs) & 81 & $39,7 \%$ \\
Tratamento químico & 55 & $27,0 \%$ \\
Tratamento com subprodutos industriais & 54 & $26,5 \%$ \\
Tratamento mecânico & 6 & $2,9 \%$ \\
Tratamento com nanopartículas & 6 & $2,9 \%$ \\
Tratamento com utilização de cimentos alternativos & 2 & $1,0 \%$ \\
\hline
\end{tabular}

Em segundo lugar estão as pesquisas que analisam o tratamento químico, com 55 publicações, empregando principalmente compostos de lítio: Nitrato de lítio ( $\left(\mathrm{LiNO}_{3}\right)$, Hidróxido de lítio (LiOH) e Carbonato de lítio $\left(\mathrm{Li}_{2} \mathrm{CO}_{3}\right)$. O principal obstáculo para o uso prático de tais adições químicas é o entendimento incompleto do mecanismo de atuação destas substâncias e os seus efeitos nas propriedades do concreto endurecido. Outro obstáculo está ligado ao fator ambiental, pois como as obras hidráulicas são as mais suscetíveis a desenvolverem RAS, existe o risco de contaminação da água e do solo. Embora o mecanismo de comportamento dos íons de lítio não seja totalmente compreendido, acredita-se que o lítio altera a composição do gel da RAS, mudando seu caráter expansivo para não expansivo, resultando em um produto com pequena ou nenhuma capacidade de absorver água e expandir. A principal aplicação dos compostos de lítio é na formação de uma camada superficial em lajes de pavimentação, placas de aeroporto, barreiras rodoviárias, pontes, visando aumentar a profundidade de penetração do composto (SILVA et al, 2010).

As pesquisas que enfocam no tratamento com subprodutos industriais somam 54 publicações e utilizam principalmente resíduos cerâmicos, agregados de concreto reciclado e resíduos de vidro. A abundante quantidade desses materiais produzidos pelas atividades industriais acaba dificultando cada vez mais o uso de aterros, pois além da produção vir aumentando nos últimos anos, os custos atuais de reprocessamento são bastante elevados, e a grande maioria desses são não biodegradáveis. Por isso, vários estudos têm sido feitos sobre a possibilidade de reaproveitar tais insumos em substituição ao agregado reativo.

Os artigos que abordam o tratamento mecânico estão relacionados com o emprego de fibras e microfibras de aço, polímeros e híbridas em estruturas suscetíveis ao fenômeno da RAS. Tais fibras podem ser adicionadas ao concreto para aumentar sua resistência à tração a fim de controlar a formação de fissuras como consequência da RAS. Esse mecanismo é importante, pois quando as forças expansivas excedem a resistência à tração surgem as fissuras, que podem resultar na redução da capacidade resistente do concreto, além de facilitar a ocorrência de outras manifestações patológicas.

Os que se referem ao uso de nanopartículas focam no aproveitamento da Nano-Sílica, Nanoargila e na combinação da nano-SiO ${ }_{2}$ e nano- $\mathrm{F}_{2} \mathrm{O}_{3}$. Atualmente, existem poucos relatos sobre a durabilidade do nanoconcreto sob a ação da RAS, mas o uso desse material tem atraído grande atenção, pois o concreto que possui nanomateriais apresenta uma melhora na durabilidade devido às propriedades físicas e químicas desses materiais, podendo melhorar a microestrutura e a composição química da solução dos poros do concreto, o que resultaria na redução da expansão proporcionada pela RAS.

Por fim, os trabalhos que abordam a utilização de cimentos alternativos concentram-se no uso do cimento de baixo teor de álcalis, de aluminato de cálcio e de sulfoaluminato de cálcio. A ideia central do uso destes cimentos seria na redução de silicatos alcalinos hidratados formados e, por consequente, redução da possibilidade de expansão no concreto.

\section{Conclusões}

Abordagem estatística da produção científica internacional sobre medidadas de mitigação da Reação Álcali-Agregado(RAA) 
A partir da análise da produção científica internacional sobre as medidas de mitigação da RAS realizada neste estudo, mediante os dados indexados nas bases SciELO, ScienceDirect, Scopus e Web of Science (WoS), sustentada pela utilização de técnicas bibliométricas, foi possível identificar um crescimento significativo no número de publicações, fato que evidencia que o aumento de casos identificados alarmou o meio técnico, e que o tema está cada vez mais difundido entre os pesquisadores. Quanto aos países que mais publicaram sobre o tema destacam-se aqueles que, historicamente, são os pioneiros em casos diagnosticados e buscam a mitigação da RAS há muitos anos, como Estados Unidos, China, Turquia e Canadá.

Após a análise e segregação dos 204 artigos selecionados, pode-se concluir que as medidas de mitigação da RAS mais pesquisadas são os tratamentos com materiais cimentícios suplementares, com adições químicas e resíduos industriais. $E$ dentre os materiais cimentícios suplementares e adições químicas mais analisados pelos artigos selecionados tem-se a cinza volante, o metacaulim, a sílica ativa e o nitrato de lítio, que já têm sido utilizados na prática durante as construções, o que evidencia que tais medidas de fato apresentam bons resultados, embora precise ter a preocupação pela busca da dosagem e materiais ideais de acordo com cada obra a fim dedirimir com eficiência os efeitos da RAS. Há que se compreender o melhor mecanismo de controle e os efeitos que tais materiais causam no concreto.

Dessa forma, pode-se concluir que as técnicas bibliométricas e a metodologia Proknow-C foram muito úteis para identificação da produção científica, e culminaram em resultados confiáveis sobre a evolução da pesquisa sobre a mitigação da Reação Álcali-Sílica. As informações presentes neste estudo podem servir como auxílio de planejamento e execução de artigos e pesquisas a fim de melhorar a compreensão da produção científica.

\section{Agradecimentos}

Gostaria de agradecer a Fundação Cearense de Apoio ao Desenvolvimento Científico e Tecnológico FUNCAP, Brasil, por disponibilizar a bolsa, na modalidade PIC-PBPU e PROVIC, e tornar possível a realização deste trabalho.

\section{Referências Bibliográficas}

AFONSO, M. H. et al. Como construir conhecimento sobre o tema de pesquisa? Aplicação do processo Proknow-C na busca de literatura sobre avaliação do desenvolvimento sustentável. Revista de Gestão Social e Ambiental, v. 5, n. 2, p. 47-62, 2011. ISSN 1981-982X.

HERNÁNDEZ-CRUZ, D. et al. Fiber reinforced mortar affected by alkali-silica reaction: $A$ study by synchrotron microtomography. Cement and Concrete Composites, v. 68, p. 123-130, 2016. ISSN 09589465.

MENÉNDEZ, E. et al. Sustainable and Durable Performance of Pozzolanic Additions to Prevent Alkali-Silica Reaction (ASR) Promoted by Aggregates with Different Reaction Rates. MDPI, p. 1-24, dez./2020.

RIBEIRO, D. et al. Estudo das reações alcalis-sílica associadas ao uso da lama vermelha em argamassas colantes e de revestimento. Cerâmica, v. 58, n. 345, p. 90-98, 2012. ISSN 0366-6913.

SILVA, D. et al. Estudo dos efeitos do LiNO3 na reação Álcali-Sílica e comparação com os efeitos da cinza volante na reação. Revista IBRACON de Estruturas e Materiais, v. 3, n. 3, p. 310-321, 2010. ISSN 19834195.

SOARES, P. B. CARNEIRO, J. C. T. CALMON, L. J. CASTRO, O. C. O. L Análise bibliométrica da produção científica brasileira sobre Tecnologia de Construção e Edificações na base de dados Web of Science. Ambiente Construído, Porto Alegre, v. 16, n. 1, p. 175-185, jan./mar. 2016

ZAPALA-SLAWETA, J.; OWSIAK, Z.; IOP. The use of lithium compounds for inhibiting alkali-aggregate reaction effects in pavement structures. Conference on Resilient and Safe Road Infrastructure (KIELCE), 2018, Kielce, POLAND. lop Publishing Ltd, May 08-09. 